\title{
SCANNING ELECTRON MICROSCOPIC AND HISTOLOGIC STUDY OF THE EFFECT OF CURCUMIN ON HEALING OF INDUCED PALATAL MUCOSAL ULCER IN ALBINO RATS
}

\author{
Mohamed Shredah* and Mona El Deeb**
}

\begin{abstract}
Introduction: Wound healing is a highly complicated event attained through precisely programmed phases. Curcumin was used as a flavoring and coloring agent. It also exhibited remarkable properties in medicinal therapy.
\end{abstract}

Aim: This study aimed to investigate the therapeutic effect of curcumin on healing of induced palatal ulcer in a rat model.

Material \& Methods: Thirty six male Albino rats were divided into three groups. Group I (Control group): rats were given the vehicle solution. Group II (ulcer induced group): ulcers were created on the hard palatal mucosa of rats of this group. Group III (curcumin \& ulcer induced group): ulcers were induced then rats were daily given $200 \mathrm{mg} / \mathrm{kg}$ of curcumin starting from the day of ulceration. The animals were sacrificed such that two rats from the control group and five rats from the experimental groups were sacrificed after three, six and twelve days post ulceration. Specimens were prepared for histological examination and scanning electron microscopic assessment.

Results: Histological examination revealed better enhancement of ulcer healing and epithelization together with establishment of more dense collagen bundles in group III samples when compared to group II throughout the examined experimental periods. Scanning electron microscopic results supported the histological findings showing significant reduction of the ulcer size in group III specimens.

Conclusion: oral administration of curcumin has beneficial effects in accelerating wound healing and improving all phases of wound repair of palatal ulcer in rats.

KEYWORDS: ulcer healing, palatal mucosa, curcumin.

* Ass. Professor, Oral Biology Department, Faculty of Dentistry, Damanhour University.

** Ass. Professor, Oral Biology Department, Faculty of Oral and Dental Medicine, Future University. 


\section{INTRODUCTION}

Oral mucosal wounds/ulcers are opened lesions which occur in the mouth due to several disorders (Gonsalves et al, 2007) ${ }^{(1)}$.

Wound healing is a normal biological process and a highly complicated event in the human body. It is attained through precisely programmed phases including inflammation, cell proliferation, wound contraction, angiogenesis, matrix remodeling and epithelialization (Wang et al, 2006 \& Guo and DiPietro 2010) ${ }^{(2,3)}$. Coordination within these phases is achieved by interactions between cells, extracellular matrix proteins, and growth factors. Any variation in this healing process will lead to abnormal healing (Wang et al, 2006) ${ }^{(2)}$.

Turmeric (Curcuma longa), is a natural herb that has been used throughout history in India and China as a spice and flavoring agent. One of its main constituents is called curcumin, which is a natural yellow pigment present in turmeric (Thangapazham et al, $2007 \&$ Prasad et al, 2014) ${ }^{(4,5)}$.

Several studies have focused on the therapeutic beneficial effects of dietary components of plant origin (Panchatcharam et al, 2006) ${ }^{(6)}$. Curcumin has been used for centuries as a spice. It also shows a great promise as a nontoxic therapeutic medication (Nasri et al, 2014) ${ }^{(7)}$.

Curcumin was used in traditional medicine in the treatment of cough, biliary disorders, rheumatism, hepatic disorders, diabetic ulcers and sinusitis (Anamika, 2012) ${ }^{(8)}$. Various studies have provided evidences concerning the possible prophylactic and therapeutic effects of curcumin regarding the antiinfectious, anti-carcinogenic, anti-apoptotic and cell proliferation activities of this compound (Yucel et al, 2011, Aktas et al, 2012 \& Qian et al, 2012) ${ }^{(9-11)}$. Curcumin has also been found to have an effective antioxidant activity (Jagetia and Aggarwal $2007 \&$ Zhang et al, 2015) ${ }^{(12,13)}$ and may lead to enhancement of wound healing (Bhagavathula et al, 2009 \& Jagetia, and Rajanikant, 2015) ${ }^{(14,15)}$. Jagetia and Rajanikant 2004,
$2005^{(16,17)}$ have reported that curcumin treatment accelerated healing of wounds in mice exposed to various doses of radiation. Moreover, it supports collagen production and decreases levels of matrix metalloproteinase (Bhagavathula et al, 2009) ${ }^{(14)}$.

Since curcumin is commonly used as a flavoring agent, and because of its valuable medicinal properties, it was thought worthwhile to investigate its therapeutic effect on healing of induced palatal ulcer in a rat model.

\section{MATERIAL AND METHODS}

\section{Experimental procedure}

Thirty six adult male Albino rats (150-200 gm) were selected for the study. The animals were housed in separate cages under the optimal experimental conditions according to the guidelines of the Animal Ethics Committee. They were fed on ground barely and supplied water add-libitum. After one week acclimatization period, the animals were divided into three groups:

Group I (Control group): Formed of six rats which were given a daily oral dose of $0.5 \%$ carboxymethylcellulose (CMC) using gastric tube as a vehicle.

Group II (ulcer induced group): Formed of fifteen rats. Prior to creation of the ulcers, the animals were anaesthetized using pentobarbital $(50 \mathrm{mg} / \mathrm{kg})$. Then, round filter papers of diameter $5.5 \mathrm{~mm}$ were saturated in $15 \mathrm{ml}$ of $50 \%$ acetic acid. In order to create round ulcer, the acid-soaked filter paper was pressed onto the hard palate of each rat for $60 \mathrm{sec}-$ onds (Fujisawa et al, 2003) ${ }^{(18)}$.

\section{Group III (curcumin \& ulcer induced group):} Formed of fifteen rats. The ulcer was induced to animals of this group similarly to group II. Then, rats were daily given $200 \mathrm{mg} / \mathrm{kg}$ of curcumin suspended in $0.5 \% \mathrm{CMC}$ delivered using gastric tube (Ghosh et al, 2009) ${ }^{(19)}$, starting from the day of ulcer induction. 
The rats were sacrificed by ketamine over dose at the end of the experimental periods. Two rats from the control group and five rats from the experimental groups were sacrificed after three, six and twelve days post ulceration. The hard palates were carefully dissected from all animals. The specimens were then prepared for routine histological study and scanning electron microscopic assessment.

\section{Light microscopic examination}

Specimens were immediately fixed in $10 \%$ neutral formalin for $48 \mathrm{~h}$. They were demineralized using a 10\% EDTA solution at PH 7.8 in a microwave oven. The demineralized specimens were then washed by tap water and embedded in paraffin. Histological sections of $5 \mu \mathrm{m}$ thickness were obtained and stained with hematoxylin and eosin stain according to the conventional method (Luna, 1968) ${ }^{(\mathbf{2 0})}$. Histological examination was performed using light microscope.

\section{Scanning electron microscopic examination (SEM) (Tahmasebi et al, 2015) (21)}

- Small pieces of fresh specimens of the palatal mucosa were removed and fixed by immersing them immediately in $4 \mathrm{~F} 1 \mathrm{G}$ (Fixative, phosphate buffer solution) $\mathrm{PH}=7.4$ at $4 \mathrm{C}$ for 3 hours.

- Specimens were then post fixed in $2 \% \mathrm{OsO} 4$ in the same buffer at $4 \mathrm{C}$ for 2 hours.

- Samples were then washed in the buffer and dehydrated at $4 \mathrm{C}$ through a graded series of ethanol.

- Samples of the palatal mucosa were dried by means of a critical point method, mounted using carbon paste on an AL-stub and coated with gold up to 400 A thickness in a sputter - coating unit (JFC-1100 E).

- Observations of the palate morphology in the coded specimens were performed in a Jeol JSM5300 scanning electron microscope operated between 15 and $20 \mathrm{KeV}$.

\section{Measuring area \% of palatal ulcers (Abramoff et al, 2004) (22)}

Image J 22 software was used for area \% measurement of the palatal ulcers. Area \% was measured in a standard measuring frame per 5 photomicrographs in each group using the available magnification of the electron microscopy transferred to the monitored screen. These areas were masked by a red binary color that could be measured by the computer system as follows:

1- Software converted the image type to 8-bit gray scale.

2- The image was then color area threshold to select only the color area of interest which represents the palatal ulcer.

3- The ulcer was then masked by a red binary to measure area fraction which is the percentage of the pixels in the ulcer.

\section{Statistical analysis}

The results of area $\%$ of the ulcer were summarized as mean and standard deviation. Results' significance was assessed by determining the probability factor ( $\mathrm{P}$ value), were $\mathrm{P} \leq 0.05$ was considered statistically significant. Data were entered and analyzed using SPSS 19 (Statistical Package for the Social Sciences). Mann-Whitney test was used to compare between different groups, Friedman test used to compare the different readings between 3, 6 and 12 days in the tested groups.

\section{RESULTS}

\section{H\&E results}

\section{Control group (group I)}

The mucosa of the hard palate of the control group demonstrated keratinized stratified squamous epithelium with uniform arrangement of epithelial layers. The epithelial cells consisted of a basal cell layer, numerous polyhedral cells forming the prickle layer, granular layer of flattened cells, and overlying 
thick keratinous layer. The epithelial rete pegs appeared short and broad while the underlying lamina propria revealed numerous fibroblasts, interlacing collagen fibers and blood vessels (Fig. 1).

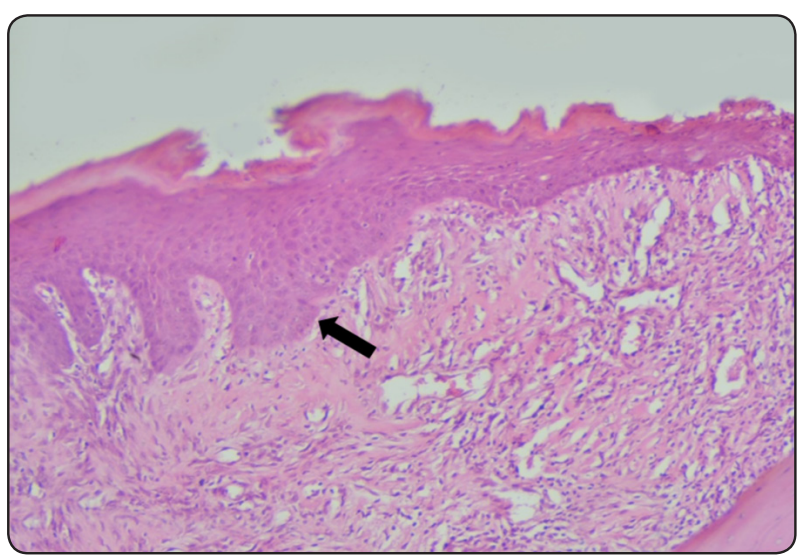

Fig. (1) Photomicrograph of rat hard palate mucosa of control group showing normal layers of keratinized stratified squamous epithelium \& underlying C.T., short \& broad epithelial rete pegs (arrow) (H\&E: 200X)

\section{Groups II \& III (3 days post ulceration)}

Histological sections from the ulcer induced group (group II) showed apparently extended part of ulceration where the ulcer edges were apart showing loss of epithelial cells. The lamina propria revealed extensive inflammatory cell infiltration filling the ulcerated area, as well as numerous dilated congested blood vessels. Marked reduction of the collagen content was also detected (Fig. $2 ; \mathrm{a}$ ). On the other hand, curcumin treated group demonstrated apparent approximation of the ulcer edges and apparent reduction in the ulcerated region which was filled with granulation tissue. Large number of inflammatory cells together with dilated engorged blood vessels were also detected throughout the lamina propria (Fig. 2;b).

\section{Groups II \& III (6 days post ulceration)}

Group II samples (6 days after ulceration) showed migration of the keratinocytes to the ulcerated area and start of re-epithelization and closure (Fig. 3;a). However, the newly formed epithelium appeared thin, with disorganized epithelial strata, vacuolated cytoplasm and detached overlying keratinous layer (Fig. 3;c). Fibroblastic proliferation and collagen fiber deposition together with scattered edematous areas could be detected within the underlying lamina propria. Numerous dilated blood vessels and inflammatory cell infiltration were also recognized (Fig. 3;a,c). On the other hand, curcumin treated group (group III) revealed apparent reduction in the extent of the ulcerated region (Fig. 3;b) with more organized epithelial layers and less vacuolization when compared to samples of group II (Fig. 3;d). Moreover, apparently less inflammatory cells and edematous areas could be noticed within the lamina propria (Fig. 3;b,d).

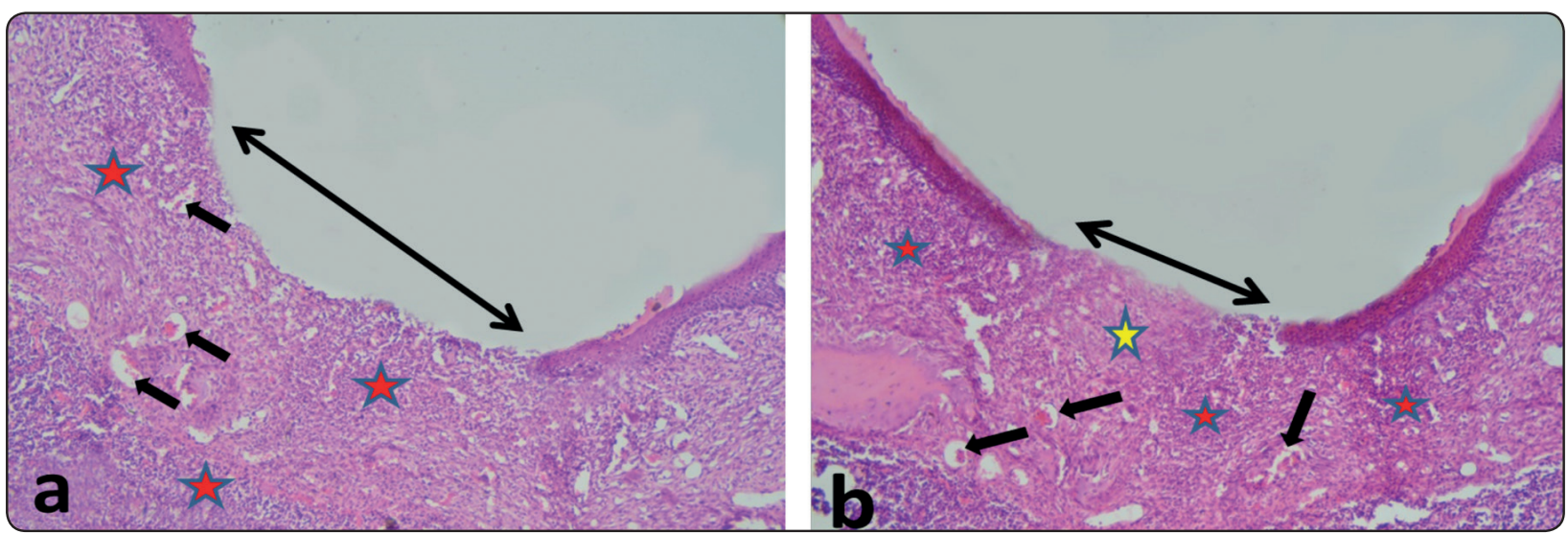

Fig. (2) Photomicrograph of rat hard palate mucosa of group II (a) and group III (b) 3 days post ulceration showing: (a): extended part of ulceration (ray), (b): marked approximation of the ulcer edges (ray), granulation tissue (yellow star), (a,b): extensive inflammatory cell infiltration (red stars), dilated and congested blood vessels (black arrows) (H\&E: (a) 200X, (b) 200X) 


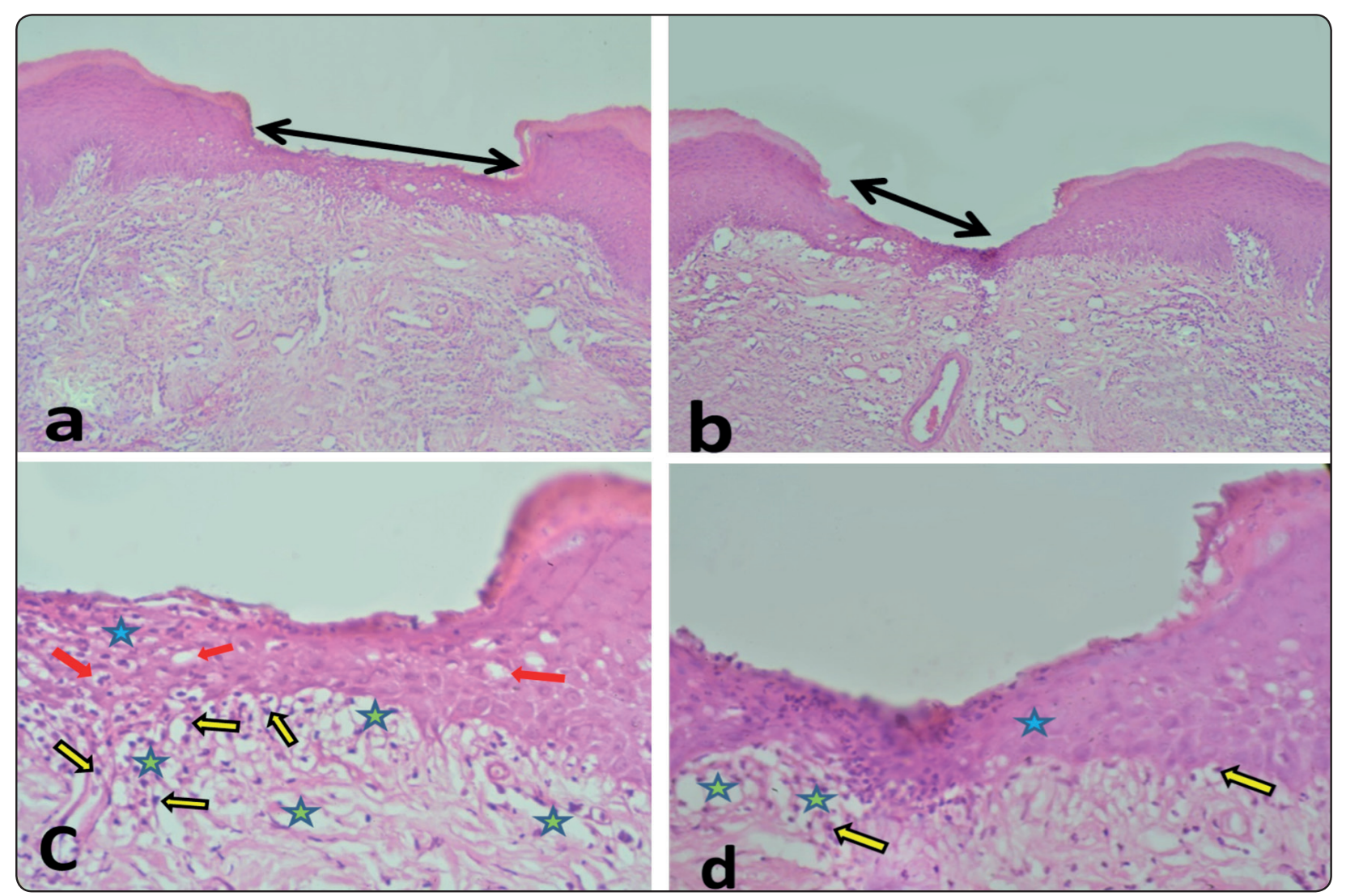

Fig. (3) Photomicrograph of rat hard palate mucosa of group II (a,c) and group III (b,d) 6 days post ulceration showing: (a): start of re-epithelization without keratinous layer (ray), (c): disorganized epithelial strata (blue star), vacuolated cytoplasm (red arrows), abundant edematous areas (green stars), inflammatory cell infiltration (yellow arrows), (b): reduction of the ulcerated region (ray), (d): more organized epithelial layers (blue star), less inflammatory cells (yellow arrows), few edematous areas (green stars) (H\&E: (a) 200X, (b) 200X, (c) 400X, (d) 400X)

\section{Groups II \& III (12 days post ulceration)}

The ulcer induced group (group II) identified continuous and thin epithelial covering of the ulcerated area without rete pegs. The lamina propria exhibited proliferating fibroblasts together with irregular collagen fiber deposition. Apparent reduction of the interstitial edema and moderate inflammatory cell infiltrate were also detectable when compared to the previous group (Fig. 4;a). On the other hand, group III specimens revealed complete healing and epithelization of the ulcer region. The epithelial covering appeared well structured with considerable thickness and more stratified strata together with rete pegs formation. There is obvious establishment of more dense, mature and organized collagen bundles and apparently more proliferating spindle shaped fibroblasts compared to group II specimens. Moreover, interstitial edema and inflammatory cells were hardly distinguished (Fig. 4;b).

\section{Scanning Electron Microscope (SEM) results}

In the current study, SEM was used to demonstrate the ulcer size in groups II and III. An obvious reduction in the ulcer size was encountered in the examined experimental periods (3, 6 and 12 days) in group III when compared to those of group II.

3 days post ulceration, group II samples revealed total deprivation of the surface epithelial covering with deep mucosal injury (Fig. 5; a). However, in group III specimens of the same interval, an obvious approximation of the ulcer edges narrowing its size was clearly shown (Fig. 5; b). 


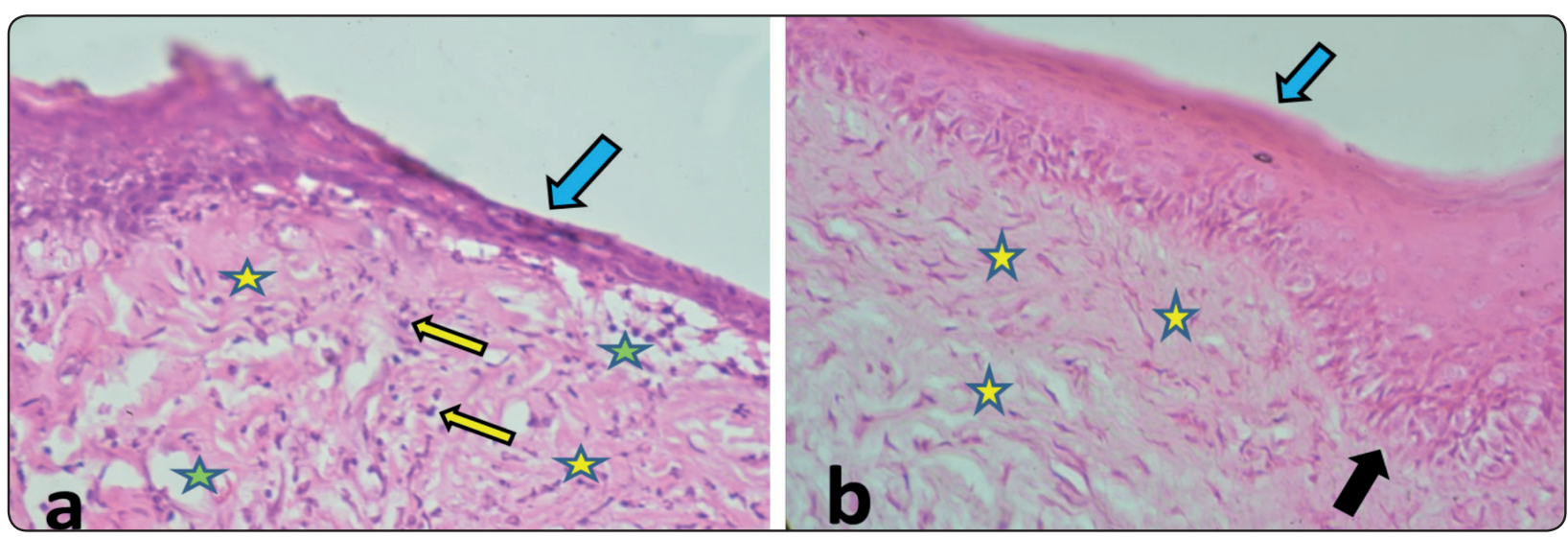

Fig. (4) Photomicrograph of rat hard palate mucosa of group II (a) and group III (b) 12 days post ulceration showing: (a): thin epithelial covering (blue arrow), irregular collagen fibers (yellow stars), few edematous areas (green stars), inflammatory cell infiltration (yellow arrows), (b): thickened \& well structured epithelial covering (blue arrow), rete pegs formation (black arrow), dense \& organized collagen bundles (yellow stars) (H\&E: (a) 400X, (b) 400X)

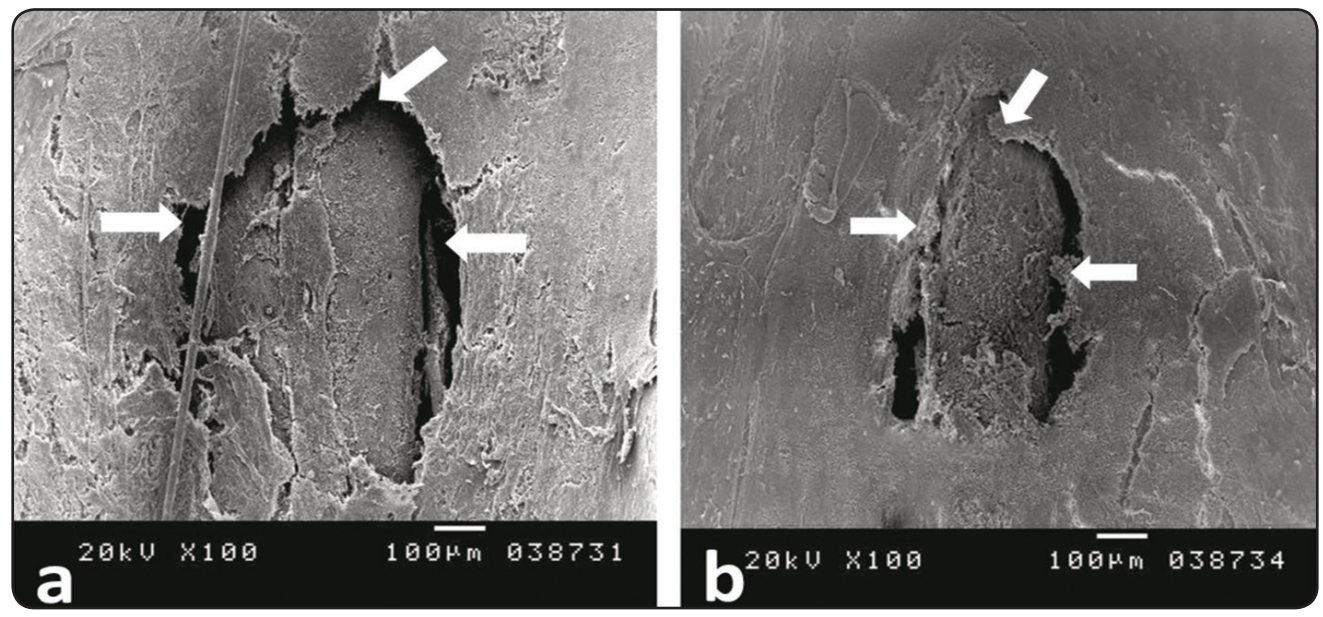

Fig. (5) Scanning electron micrograph of rat hard palate mucosa of group II (a) and group III (b) 3 days post ulceration showing: (a): detached epithelial surface covering. (b): obvious approximation of the ulcer edges (a) 100X, (b) 100X

At 6 days post ulceration, a shallow ulcer was formed with an attempt to repair the injury by covering the ulcer. Group III specimens revealed better enhancement of ulcer healing when compared to group II of the same interval (Fig. 6; a,b). Few collagen fibers were deposited within the center and depth of the ulcerated area in group II (Fig. 7; a). The appearance of an epithelial bridge at the ulcer margins together with establishment of a mesh of fiber bundles within the ulcer base were markedly spotted in group III sections (Fig. 7; b).

12 days post ulceration, group II samples exhibited small ulcer size with clearly detected ulcer zone (Fig. 8; a). On the other hand, group III showed that the ulcer area has almost disappeared with difficulty to identify its original site (Fig. 8; b). 


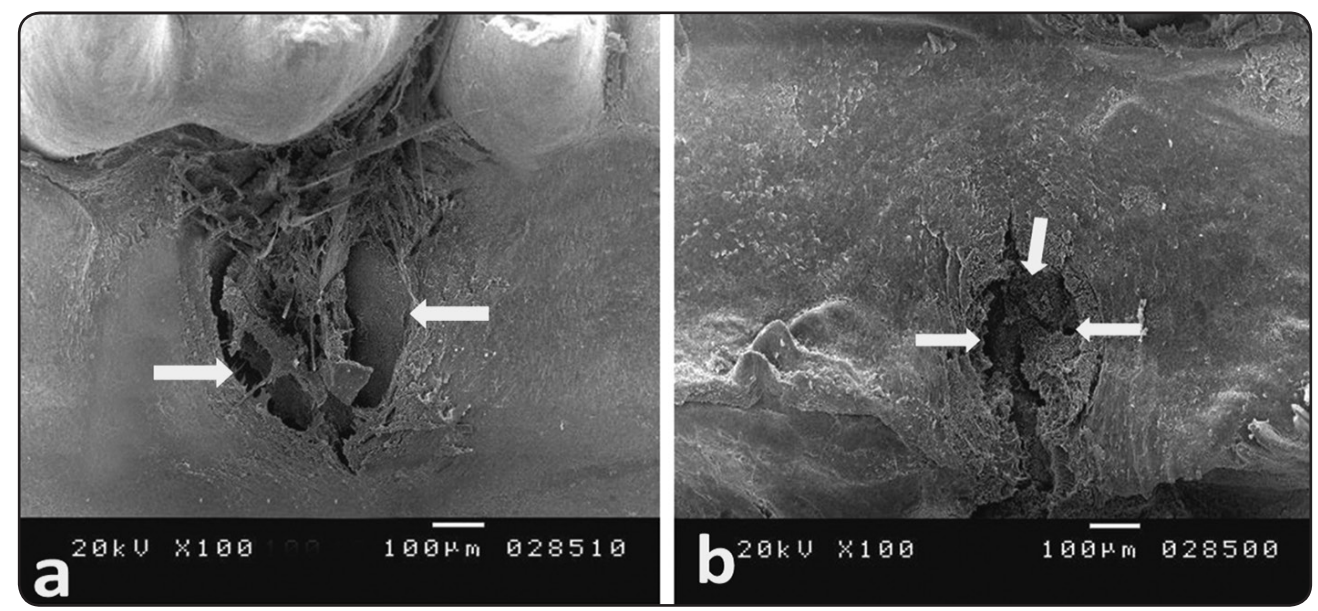

Fig. (6) Scanning electron micrograph of rat hard palate mucosa of group II (a) and group III (b) 6 days post ulceration showing: evident reduction of the ulcer size in group III than group II (a) 100X, (b) $100 \mathrm{X}$

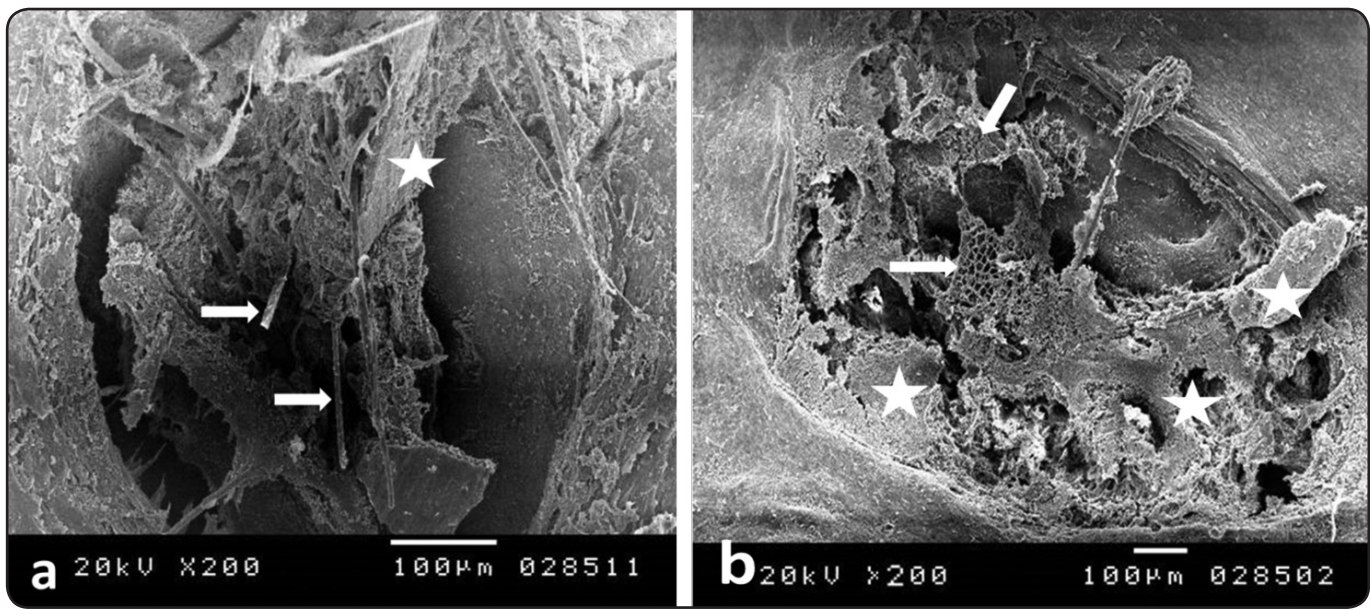

Fig. (7) Higher magnification of rat hard palate mucosa of group II (a) and group III (b) 6 days post ulceration showing: (a): area of epithelial covering (star), few collagen fibers (arrows), (b): epithelial bridges at the ulcer margins (stars), mesh of fiber bundles (arrows) (a) 200X, (b) 200X

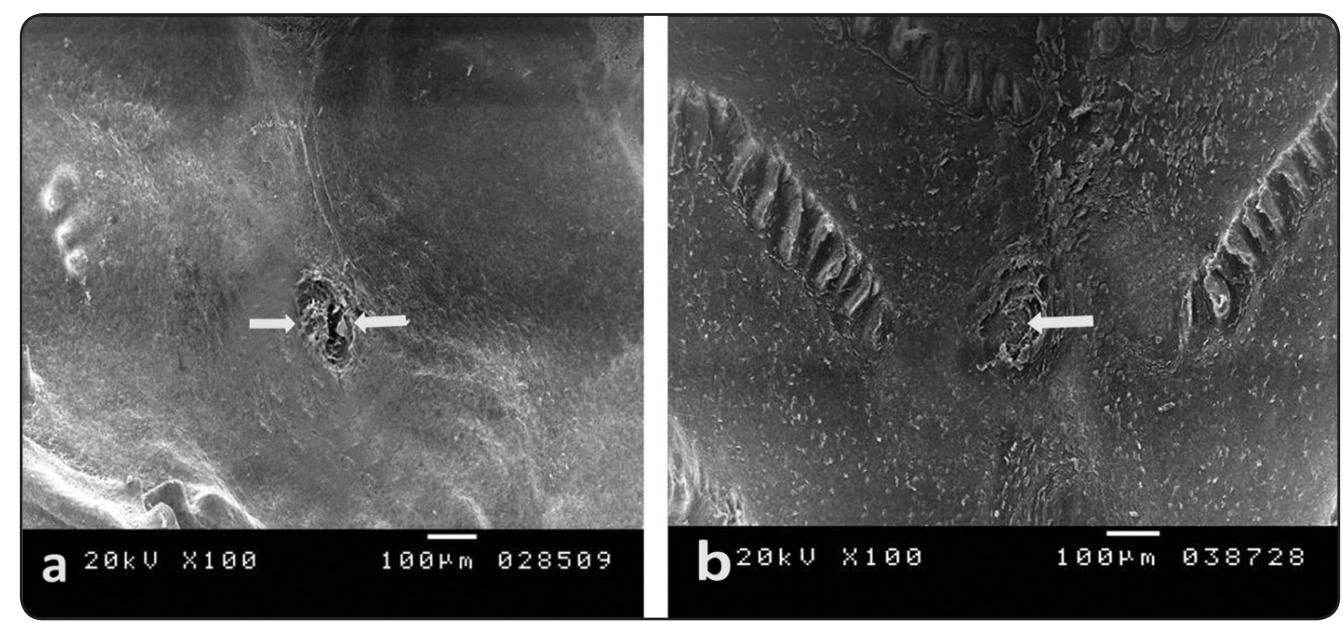

Fig. (8) Scanning electron micrograph of rat hard palate mucosa of group II (a) and group III (b) 12 days post ulceration showing: (a): small ulcer size. (b): almost disappeared ulcer area (a) 100X, (b) 100X 


\section{Image analysis and statistical results}

On measuring the area $\%$ of the palatal ulcers in the experimental groups, P-value and mean $\pm \mathrm{SD}$ results revealed significant reduction in the area \% between groups II and III during 3 and 6 days post ulceration, while the difference between the tested groups within the $12^{\text {th }}$ day did not reach the level of significance. Moreover, statistically significant increase in the healing of palatal ulcers was detected during different durations of the experiment (3, 6, 12 days) within each tested group (II and III) (table 1, figure 9).

TABLE (1): Comparison of area \% between groups II and III during different durations

\begin{tabular}{|c|c|c|c|}
\hline Area \% & Group II & Group III & P \\
\hline 3 days & $45.9 \pm 1.06$ & $29.2 \pm 1.8$ & $0.001^{*}$ \\
\hline 6 days & $23.3 \pm 1.5$ & $20.1 \pm 1.8$ & $0.01 *$ \\
\hline 12 days & $17.5 \pm 1.9$ & $14.5 \pm 0.2$ & 0.1 \\
\hline $\mathbf{P}$ & $0.02 *$ & $0.001 *$ & \\
\hline
\end{tabular}

* significant difference

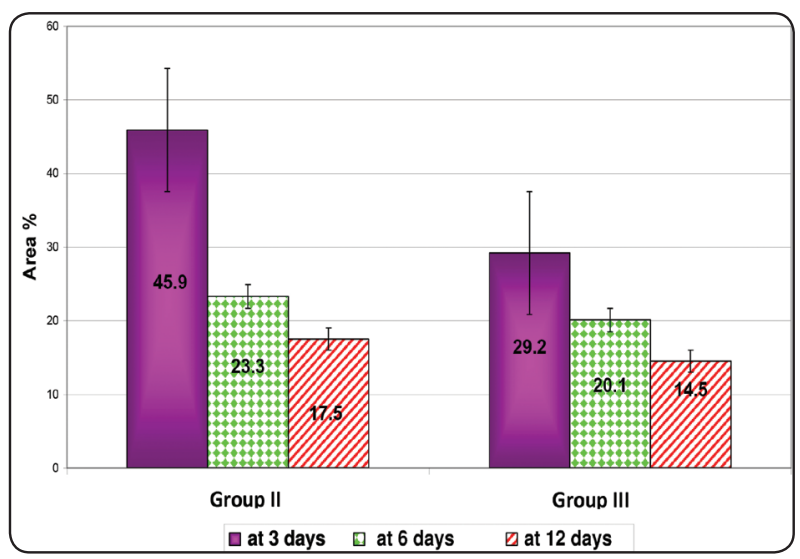

Fig. (9) Mean values of area \% between groups II and III during different durations.

\section{DISCUSSION}

Wound healing and tissue repair are complicated processes involving inflammation, angiogenesis, fibroplasia, wound contraction, and epithelization of the wound defect. The wound healing process is necessary to reform the damaged tissue and to inhibit its invasion by pathogens. The healing cascade starts immediately following the injury and last for variable periods of time depending on the degree of wounding (Panchatcharam et al, 2006) ${ }^{(6)}$.

Curcumin has been widely used as a flavoring and coloring agent. It also exhibited remarkable properties in medicinal therapy. The absence of toxicity of curcumin, even when given at high doses, together with its various biological properties makes it important to explore its use in several disorders (Muhammad, N. and Ghani, 2015) ${ }^{(23)}$.

In $\mathrm{H} \& \mathrm{E}$ results of the present study, the ulcer induced group (group II) showed apart ulcer edges 3 days post ulceration. Newly formed epithelium with disorganized epithelial strata appeared at 6 days duration till formation of thin epithelial covering over the ulcerated area after 12 days. Curcumin treated group (group III) revealed marked approximation of the ulcer edges and formation of more organized epithelial layers reaching to complete epithelization with well structured and thickened epithelial strata throughout the three experimental duration periods. Better enhancement of ulcer healing and approximation of ulcer edges of curcumin treated group was also supported by scanning electron microscopic findings. Same results were obtained by Panchatcharam et al, $2006{ }^{(6)}$ who reported that curcumin reduced the epithelialization period compared to the control group when topically applied to wounds in a rat model. They added that optimal re-epithelialization was observed after 12 days of continued curcumin treatment. Moreover, hairless rats topically pretreated with curcumin followed by corticosteroid 
treatment and induction of superficial abrasion skin wound revealed slight thickening of the epidermis and rapid skin healing (Bhagavathula et al, 2009(14). Another study by Xingyi et al, $2012{ }^{(24)}$ showed that re-epithelialization of wounds occurred on the 7th day when curcumin was applied to full thickness excisional wounds. It was also observed that curcumin treated burn wounds in rats' skin heal much faster than burn wounds only. Squamous epithelia started to re-epithelialize on the 4th day, also rise in the expression of proliferating cell nuclear antigen was detected indicating high rate of cellular proliferation (Kulac et al, 2013) ${ }^{(25)}$. Lastly, the impact of curcumin on oral ulcer healing in rats' tongue was studied by Zaher et al, $2014{ }^{(26)}$ who demonstrated marked migration of the epithelium at the 6th day and formation of organized epithelial covering at the 12 th day post ulceration.

It is worth noting that $\mathrm{H} \& \mathrm{E}$ results of both experimental groups (II \& III) 6 days post ulceration showed complete re-epithelization and closure of the ulcerated area. However, scanning electron microscopic results of the same interval revealed appearance of epithelial bridge without complete closure of the ulcer. This conflict of results can be explained as scanning electron microscope studies the whole exposed surface area of the ulcer, while H\&E sections represent only cutting sections through the ulcerated area without exposing the whole outer surface. Therefore, H\&E sections may show complete epithelization and covering of the ulcer, but actually areas without overlying covering epithelium still exist and appear using scanning electron microscopic study.

Oxidative stress is a major factor in the process of wound healing and generally prevents tissue remodeling (Thangapazham et al., 2013) (27). Curcumin was found to exert an antiulcer activity via its ability to induce glutathione-S-transferase which hinders generation of free radicals and acts as a free radical scavenger and antioxidant, leading to prevention of lipid peroxidation (Jelveh et al, 2013) ${ }^{(28)}$. It was also reported that the diferuloylmethane part of curcumin exerts antioxidant properties, comparable to vitamins $\mathrm{C}$ and $\mathrm{E}$, without causing toxic effect even at high doses (Guimarães et al, 2011) ${ }^{(29)}$. Its direct antioxidant action is mediated by regulation of some enzymes, inflammatory cytokines and growth factors (Haghighi et al, 2013) ${ }^{(30)}$. Curcumin treatment before irradiation of mouse skin resulted in rise in the concentration of glutathione as well as the activities of both superoxide dismutase and glutathione peroxidase enzymes, whereas lipid peroxidation dropped significantly. Curcumin was thought to lower lipid peroxidation by keeping the activities of glutathione and antioxidant enzymes at higher levels, as these enzymes have a significant role in the regulation of lipid peroxidation (Jagetia, and Rajanikant, 2015) ${ }^{(15)}$.

In the herein study, 3 days post ulceration demonstrated extensive inflammatory cell infiltration with numerous dilated blood vessels and marked reduction of the collagen content in groups II and III. Results concerning the inflammatory cell infiltrate were coincident with Xingyi et al, $2012^{(24)}$ and Kulac et al, $2013^{(25)}$ who reported an increase in inflammatory cells infiltration in granulation tissue on the 3rd day of curcumin treatment in a full thickness excisional wounds and burn wounds respectively. One possible explanation was proposed by Bernatchez et al., $2013{ }^{(31)}$ who verified that curcumin enhanced nitric oxide (NO) production in excisional wounds of mice exposed to gamma radiation. Jagetia and Rajanikant, $2012{ }^{(32)}$ and Bernatchez et al., $2013{ }^{(31)}$ concluded that curcumin treatment increased NO production which helps wound healing by promoting inflammation. Although our study suggested that enhancing the natural inflammatory response caused by curcumin improved wound healing, some papers proposed that curcumin actually reduces inflammation (Akbik et al, 2014) ${ }^{(33)}$. 
It was obvious that the inflammatory cells decreased with time throughout the healing intervals in the curcumin group more apparently than the ulcer induced one, which was concomitant with Muhammad, N. and Ghani, $2015^{(23)}$. It is well acknowledged that reducing the inflammatory response allows the tissue to enter the following stages of healing such as proliferation and remodeling. Uncontrolled and persistent inflammation retards the later stages of healing and thus delays the wound healing process (Akbik et al, 2014) ${ }^{(33)}$.

Our data demonstrated that fibroblastic proliferation and collagen fiber deposition started to appear after 6 days of ulceration in groups II and III of $\mathrm{H} \& \mathrm{E}$ sections. 12 days post ulceration, group III showed establishment of more dense, mature and organized collagen bundles and apparently more proliferating fibroblasts compared to group II specimens. This was supported by the scanning electron microscopic results which revealed establishment of a mesh of fiber bundles within the ulcer base in group III sections. It is crucial to report that the infiltration of fibroblasts into the wound site is necessary for collagen production and deposition (Loughlin and Artlett, 2011) ${ }^{(34)}$, and collagen formation is essential for enhancing wound repair (Akbik et al, 2014) ${ }^{(33)}$. This finding was parallel to that documented by Mohanty et al, 2012, Zaher et al, 2014 and Mehrabani et al, $2015^{(35,26,36)}$ who postulated that curcumin treatment lessens wound healing time, promotes collagen deposition and increases fibroblastic proliferation. They added that the resulting collagen is well-aligned and more compacted, and the bundles appeared to be thicker in the curcumin treated wound biopsy specimens when compared to the untreated samples. In this regard, it is conceivable to mention that transforming growth factor $\beta 1$ (TGF- $\beta 1$ ) is essential in wound healing as it enhances fibronectin and collagen expression by fibroblasts and raises the rate of granulation tissue synthesis in vivo. Based on these data, it was found that curcumin treatment increased the expression of
TGF- $\beta 1$ resulting in stimulation of fibronectin and collagen deposition (Maheshwari et al, 2006) ${ }^{(37)}$. Contrastingly, in an in vitro wound healing model (by scratching a line within the cell layer), curcumin showed no effect on the fibroblastic migration to the wound site (scratch line). This contradictory result may be attributed to the difficulty in effectively imitating the complex wound healing process which occurs in an in vitro setting. (Topman et al., 2013) ${ }^{(38)}$.

In the current work, $\mathrm{H} \& \mathrm{E}$ stained sections showed apparently less edematous areas in the curcumin treated group 6 days post ulceration which became hardly distinguished in 12 days specimens. This forgoing observation was in close agreement with Topcu-Tarladacalisir et al, $\mathbf{2 0 1 3}^{(39)}$ whorevealed that oral curcumin pretreatment clearly improved the abnormal histological finding of edematous lamina propria in acetic acid induced ulcerative colitis in rats.

Image analysis results revealed significant reduction of the area \% between groups II and III during 3 and 6 days post ulceration. Moreover, statistically significant increase in the healing of palatal ulcers was detected throughout the experimental periods (3, 6, 12 days) within each tested group (II and III), which was compatible with the histological H \& E observations. This obtained data was in close agreement with Durgaprasad et al, 2011 and Mehrabani et al, $2015^{(40,36)}$ who postulated that administration of curcumin on the back skin of a burn wound in rats showed significant increase in wound contraction percentage and reduction in its size. Also in a randomized placebo-controlled clinical trial, curcumin gel showed noticeable decrease in the size of aphthous ulcer within the 4th and 7th days of the trial (Manifar et al, 2012) ${ }^{(41)}$. It is conceivable to suggest that during granulation tissue formation, fibroblasts differentiate into myofibroblasts as contraction progresses and resistance raises. The presence of myofibroblasts is a characteristic feature 
of tissue undergoing contraction. Fast wound contraction in curcumin-treated wounds may be partly related to the apparent increase of myofibroblasts (Panchatcharam et al, 2006) ${ }^{(6)}$.

In summary, our study substantiates that the oral administration of curcumin has beneficial effects in accelerating wound healing and improving all phases of wound repair of palatal ulcer in rats, including epithelialization, wound contraction and collagen synthesis resulting in reduction of the ulcer size. Curcumin may therefore be a viable alternative or an adjuvant therapy to standard existing drugs used for treatment of oral ulcers.

\section{REFERENCES}

1- Gonsalves, W., Chi, A. and Neville, B.: Common oral lesions: Part I. Superficial mucosal lesions. Am. Fam. Physician., 75: 501-507, 2007.

2- Wang, J., Boerma, M., Fu, Q. and Hauer-Jensen, M.: Radiation responses in skin and connective tissues: effect on wound healing and surgical outcome. Hernia, 10:502-506, 2006.

3- Guo, S. and DiPietro, L.: Factors affecting wound healing. J. Dent. Res., 89: 219-229, 2010.

4- Thangapazham, R., Sharma, A. and Maheshwari, R.: Beneficial role of curcumin in skin diseases. Adv. Exp. Med. Biol., 595: 343-57, 2007.

5- Prasad, S., Gupta, S., Tyagi, A. and Aggarwal, B.: Curcumin, a component of golden spice: from bedside to bench and back. Biotechnol., Adv., 32: 1053-1064, 2014.

6- Panchatcharam, M., Miriyala, S., Gayathri, V. and Suguna, L.: Curcumin improves wound healing by modulating collagen and decreasing reactive oxygen species. Molecular and Cellular Biochemistry, 290: 87-96, 2006.

7- Nasri, H., Sahinfard, N., Rafieian, M., Rafieian, S., Shirzad, M. and Rafieian-kopaei, M.: Turmeric: A spice with multifunctional medicinal properties. J. Herb. Med. Pharmacol., 3: 5-8, 2014.

8- Anamika, B.: Extraction of curcumin. J. Environ. Sci. Toxicol. Food Technol., 1:1-16, 2012.

9- Yucel, A., Kanter, M., Pergel, A., Erboqa, M. and Guzel, A.: The role of curcumin on intestinal oxidative stress, cell proliferation and apoptosis after ischemia/reperfusion injury in rats. J. Mol. Histol., 42: 579-587, 2011.
10- Aktas, C., Kanter, M. and Kocak, Z.: Antiapoptotic and proliferative activity of curcumin on ovarian follicles in mice exposed to whole body ionizing radiation. Toxicol. Ind. Health, 28: 853-864, $2012 \mathrm{~b}$.

11- Qian, J., Zhai, X., Niu, M., Zhou, Q. and Zhou, Y.: Curcumin inhibits iron overload-induced hepatocytic apoptosis and nuclear factor-jB activity. Zhonghua Yi Xue Za Zhi., 92: 1997-2001, 2012.

12- Jagetia, G. and Aggarwal, B.: "Spicing up" of the immune system by curcumin. J. Clin. Immunol., 27: 19-35, 2007.

13- Zhang, N., Li, H., Jia, J. and He, M.: Anti-inflammatory effect of curcumin on mast cell-mediated allergic responses in ovalbumin-induced allergic rhinitis mouse. Cell Immunol., 298: 88-95, 2015.

14- Bhagavathula, N., Warner, R., DaSilva, M., McClintock, S., Barron, A., Aslam, M., Johnson, K. and Varani, J.: A combination of curcumin and ginger extract improves abrasion wound healing in corticosteroid-impaired hairless rat skin. Wound Repair Regen., 17: 360-6, 2009.

15- Jagetia, G. and Rajanikant, G.: Curcumin Stimulates the Antioxidant Mechanisms in Mouse Skin Exposed to Fractionated $\gamma$-Irradiation. Antioxidants, 4: 25-41, 2015.

16- Jagetia, G. and Rajanikant, G.: Effect of various doses of curcumin on the radiation-impaired healing of excision wound in mice: a preliminary study. J. Wound Care, 13: 107-109, 2004a.

17- Jagetia, G. and Rajanikant, G.: Curcumin treatment enhances the repair and regeneration of wounds in mice hemi-body exposed to c radiation. Plast. Reconstr. Surg., 115: 515-528, 2005.

18- Fujisawa, K., Miyamoto, Y. and Nagayama, M.: Basic fibroblast growth factor and epidermal growth factor reverse impaired ulcer healing of the rabbit oral mucosa. J. Oral Pathol. Med., 32: 358-366, 2003.

19- Ghosh, S., Massey, H., Krieg, R., Fazelbhoy, Z., Ghosh, S., Sica, D., Fakhry, I. and Gehr, T.: Curcumin ameliorates renal failure in 5/6 nephrectomized rats: role of inflammation. Am. J. Physiol. Renal. Physiol., 296: F1146-F1157, 2009.

20- Luna, H.: Manual of histologic staining methods of the armed forces institute of pathology. [ed.] Luna L.G. 3rd. New York: Mc Graw-Hill, P.70, 1968.

21- Tahmasebi, P., Javadpour, F. and Sahimi, M.: Three-Dimensional Stochastic Characterization of Shale SEM Images. Transport in Porous Media, 110: 521-531, 2015. 
22- Abramoff, M., Magalhaes, P. and Ram, S.: Image Processing with Image J. Biophotonics International, 11: 36-42, 2004.

23- Muhammad, N. and Ghani, B.: Histological evaluation of the effect of topical application of Curcumin powder and essential oil on skin wound healing. J. Bagh. College Dentistry, 27: 58-63, 2015.

24- Xingyi, L., Kaihui, N., Lingli, L., Zhaoliang, Z. and Hao, C.: In vivo evaluation of curcumin nanoformulation loaded methoxy poly (ethylene glycol)-graft-chitosan composite film for wound healing application. Carbohydrate Polymers, 88: 84-90, 2012.

25- Kulac, M., Aktas, C., Tulubas, F., Uygur, R., Kanter, M., Erboga, M., Ceber, M., Topcu, B. and Ozen, O.: The effects of topical treatment with curcumin on burn wound healing in rats. J. Mol. Hist., 44: 83-90, 2013.

26- Zaher, A., Elsabaa, H., Abou Elkhier, M. and Elhindawy, M.: Impact of Curcumin on Tongue Ulcer Healing in Albino Rats. Mansoura Journal of Dentistry, 1: 85-89, 2014.

27- Thangapazham, R., Sharad, S. and Maheshwari, R.: Skin regenerative potentials of curcumin. Biofactors, 39: 141-9, 2013.

28- Jelveh, S., Kaspler, P., Bhogal, N., Mahmood, J., Lindsay, P., Okunieff, P., Doctrow, S.R., Bristow, R. and Hill, R.: Investigations of antioxidant-mediated protection and mitigation of radiation-induced DNA damage and lipid peroxidation in murine skin. Int. J. Radiat. Biol., 89: 618$627,2013$.

29- Guimarães, M., Coimbra, L., Aquino, S., Spolidorio, L., Kirkwood, K. and Junior, C.: Potent anti-inflammatory effects of systemically-administered curcumin modulates periodontal disease in vivo. J. Periodontal Res., 46: 2692792011

30- Haghighi, N., Naghsh, N. and Mehrabani, D.: The protective effect of curcuma longa in thioacetamide-induced hepatic injury in rat. Global J. Pharmacol., 7: 203-7, 2013.

31- Bernatchez. S., Menon, V., Stoffel, J., Walters, S., Lindroos, W., Crossland, M.: Nitric oxide levels in wound fluid may reflect the healing trajectory. Wound Repair Regen., 21: 410-7, 2013.
32- Jagetia, G. and Rajanikant, G.: Acceleration of wound repair by curcumin in the excision wound of mice exposed to different doses of fractionated gamma radiation. Int. Wound J., 9: 76-92, 2012.

33- Akbik, D., Ghadiri, M., Chrzanowski, W. and Rohanizadeh, R.: Curcumin as a wound healing agent. Life Sciences, 116: 1-7, 2014

34- Loughlin,D. and Artlett,C.: Modification of collagen by 3-deoxyglucosone alters wound healing through differential regulation of p38 MAP kinase. PLOS One, 6: e18676, 2011.

35- Mohanty, C., Das, M. and Sahoo, S.: Sustained wound healing activity of curcumin loaded oleic acid based polymeric bandage in a rat model. Mol. Pharm., 9: 2801-11, 2012.

36- Mehrabani, D., Farjam, M., Geramizadeh, B., Tanideh, N., Amini, M. and Panjehshahin, M.: The Healing Effect of Curcumin on Burn Wounds in Rat. World J. Plast. Surg., 4: 29-35, 2015.

37- Maheshwari, R., Singh, A., Gaddipati, J. and Srimal, RC. Multiple biological activities of curcumin: a short review. Life Sci., 78: 2081-7, 2006.

38- Topman, G., Lin, F. and Gefen, A.: The natural medications for wound healing-curcumin, aloevera and ginger-do not induce a significant effect on the migration kinematics of cultured fibroblasts. J. Biomech., 46: 170-4, 2013.

39- Topcu-Tarladacalisir, Y., Akpolat, M., Uz, Y., Kizilay, G., Sapmaz-Metin, M., Cerkezkayabekir, A. and Omurlu, I.: Effects of Curcumin on Apoptosis and Oxidoinflammatory Regulation in a Rat Model of Acetic Acid-Induced Colitis: The Roles of c-Jun N-Terminal Kinase and p38 MitogenActivated Protein Kinase. J. Med. Food, 16: 296-305, 2013.

40- Durgaprasad, S., Reetesh, R., Hareesh, K. and Rajput, R.: Effect of a topical curcumin preparation (BIOCURCUM$\mathrm{AX})$ on burn wound healing in rats. JPBMS, 8: 1-3, 2011.

41- Manifar, S., Obwaller, A., Gharehgozloo, A., Boorboor, H. and Akhondzadeh, S.: Curcumin Gel in the Treatment of Minor Aphthous Ulcer: Randomized, Placebo- Controlled Trial. Journal of Medicinal Plants, 41: 40-45, 2012. 